\title{
Impacto de la crisis en las cooperativas de vino: desempeño y estrategias en Castilla-La Mancha
}

\author{
J.S. Castillo-Valero ${ }^{1, *}$, K. Simon-Elorz² y M.C. García-Cortijo ${ }^{1}$ \\ 1 Instituto de Desarrollo Regional, Universidad de Castilla-La Mancha, Campus Universitario, s/n, 02071, \\ Albacete, España \\ 2 Departamento de Gestión de Empresas, Universidad Pública de Navarra, Campus Arrosadía, s/n, 31006, \\ Pamplona. España
}

\section{Resumen}

El objetivo de este trabajo es el estudio del desempeño, estrategias y posibilidades de adaptación de las cooperativas del vino en Castilla La-Mancha, comparando un periodo de expansión económica (20022006) con un periodo de crisis (2007-2011). Para ello se define un modelo de panel en el que se analiza la relación entre el desempeño económico y diferentes estrategias de gestión (del entorno, comerciales y de financiación). También se analiza el efecto de la actuación pública, en este contexto, valorando el impacto que las Ayudas del Fondo Europeo Agrícola de Desarrollo Rural 2007-2013, en promoción de las inversiones de modernización, han tenido sobre los resultados de las cooperativas. Los resultados muestran que el sector cooperativo del vino de Castilla-La Mancha ha demostrado su robustez y capacidad de adaptación en la actual crisis económica y financiera y que estas entidades de carácter social, se han adaptado mejor y más consistentemente a la nueva situación que las sociedades capitalistas, tras comparar los resultados con los obtenidos en otros trabajos. Así, las cooperativas muestran su viabilidad social y economía como modelo empresarial idiosincrático.

Palabras clave: Bodegas cooperativas, desempeño, crisis económica, estrategias.

\begin{abstract}
Impact of the crisis period on the coop-wineries: performance and strategies in Castilla La Mancha

The aim of this paper is to study the performance, strategies and adaptability of the coop-wineries in Castilla-La-Mancha between the periods of growth economic (2002-2006) and the crisis (2007-2011). We have defined a Panel Model to analyze the impact on the economic performance the different managerial decisions (environmental, commercial and financial). Also we have considered the effect of public performance of amounts from the European Agricultural Fund for Rural Development 2007-2013, on the modernization of investments and the results for the coop-wineries. The results show that the cooperative sector Wine Castilla-La Mancha has displayed its robustness and resilience in the current economic and financial crisis. The cooperatives have been better and more consistently adapted to the new situation, showing social viability and economics as idiosyncratic business model.
\end{abstract}

Key words: Coop-wineries, performance, crisis period, strategies.

* Autor para correspondencia: sebastian.castillo@uclm.es http://dx.doi.org/10.12706/itea.2015.012 


\section{Introducción}

Las cooperativas juegan un papel fundamental en la economía agraria y en el mundo rural. Este tipo de sociedades posibilitan la viabilidad de muchas explotaciones agrarias, mejoran la eficiencia de otras, incrementan el valor añadido de las producciones, crean puestos de trabajo y facilitan el acceso a los mercados de los productos agrícolas. Además, las cooperativas abastecen a la población de alimentos de calidad y contribuyen al desarrollo y cohesión social del medio rural en la medida que mejoran la renta de los agricultores y fijan la población al territorio (Arcas-Lario et al., 2013). En este contexto, la viabilidad de las cooperativas, actúa como motor de desarrollo regional, de ahí la importancia de un sector cooperativo solvente y que garantice la transmisión de rentas a los socios cooperativistas.

El ciclo económico, está poniendo a prueba la supervivencia y continuidad de las empresas, por lo que el estudio del sector cooperativo cobra especial relevancia en este momento de crisis. El sector cooperativo en Castilla-La Mancha, tiene una importante presencia en la actividad regional y, es el sector vitivinícola, uno de los más importantes en generación de actividad económica.

El último informe de Cooperativas Agro-alimentarias (2014) publica una serie de datos que evidencian la importancia del sector cooperativo en Castilla-La Mancha y que, ahondando en el sector vitivinícola, corroboran que es la región más importante de España en producción y facturación de vino. El estudio constata que Castilla-La Mancha es la primera Comunidad Autónoma en facturación de mostos y vinos a granel en empresas cooperativas, y que sólo esta región aglutina más del $50 \%$ de la facturación nacional de vinos y mostos cooperativizada. Por eso, las cooperativas de vino son fundamentales en el desarrollo del sector (Baamonde, 2009) y su desempeño económico juega un papel básico en el crecimiento de estas sociedades, sobre todo en la actual crisis, que les supone un duro reto para mantener sus cuentas saneadas y sobrevivir a las circunstancias (Carmona et al., 2013) y con un proceso selectivo donde sólo se mantendrán las más eficientes (Baamonde, 2009).

La importancia de las cooperativas agrarias ha hecho que en los últimos años se venga prestando una creciente atención al estudio de su desempeño. Vargas y García (2003) proponen un paquete de indicadores para medir el desempeño en un conjunto de 32 sociedades cooperativas de la provincia de Huelva y Jaén. Díaz-Barceló (2007) realiza un análisis financiero comparado de cooperativas vitivinícolas de Castilla La Mancha con las empresas de los principales países productores de la Unión Europea. Guzmán et al. (2009) estudian el desempeño de 81 y 106 sociedades italianas y españolas, respectivamente. Carmona et al. (2013) analiza la evolución económico-financiera de las sociedades cooperativas españolas en los años 2004 (bonanza) y 2007 (crisis). Kalogeras et al. (2013) evalúan el éxito financiero de las cooperativas agroalimentarias holandesas para el período 1999-2010.

El objetivo de esta investigación es analizar la relación entre las variables, del entorno, comerciales y de financiación, y las medidas de desempeño en las bodegas cooperativas de Castilla La Mancha. Además, se compararán las estrategias seguidas en la etapa de crecimiento económico (2002-2006) y en la de recesión (2007-2011).

La hipótesis principal (HP) analizada en este trabajo afirma:

HP: El desempeño económico de las cooperativas de vino es función de

HP1: Variables de entorno

HP2: Estrategias comerciales

HP3: Estructura financiera

HP4: Ayudas Públicas 


\section{Material y métodos}

El sector cooperativo del vino en Castilla-La Mancha

La elección de Castilla La Mancha como objeto de estudio, es consecuencia de la relevancia que esta región tiene como productora de vino a nivel mundial, así como por el peso que las cooperativas tienen en la producción vitivinícola. En el año 2013, el sector cooperativo ha representado el $67 \%$ de la producción de vino de la región, por lo que su estudio cobra especial relevancia para comprender las estrategias del sector.

El sector vitivinícola ha sido uno de los más dinámicos de los últimos años, con unas inversiones importantes por parte de las bodegas, acogidas a las ayudas de la PAC y de los viticultores invirtiendo en la reconversión y reestructuración de los viñedos (JCCM, 2014). Además, más de 700 industrias agroalimentarias están relacionadas con la producción vitivinícola lo que pone de manifiesto, la importante fortaleza económica del sector y la mejora de la rentabilidad de las explotaciones (Ruíz, 2013).

La región de Castilla-La Mancha es la mayor superficie de viñedo del mundo, así como su territorio es el primer productor global. En términos comparativos con España, la región de Castilla-La Mancha representa casi el $47 \%$ de todo el viñedo del país, y más de $48 \%$ de su producción de vino y mosto, como puede observarse en la Tabla 1. Por tanto, está plenamente justificada la elección de esta región como estudio de caso.

En Castilla-La Mancha operan 218 cooperativas con producción en vinos y mostos o derivados de los mismos; de éstas, 164 son cooperativas de Primer Grado, 4 de Segundo Grado y 15 SAT (Sociedad Agraria de Transformación). De los datos que aparecen en la
Tabla 2, se desprende que las cooperativas representa una parte muy significativa de predominio en el nivel nacional. Representan el $35 \%$ de las cooperativas y el $50,7 \%$ de la facturación (cooperativas de primer grado y SAT) respecto al país. Por secciones productivas, el $95 \%$ de la facturación es de vino a granel y mostos, situando a Castilla-La Mancha como la primera Comunidad Autónoma en estos productos.

La facturación de las cooperativas de segundo grado es de 53,09 millones de euros. Así, en total, las cooperativas de vino de Castilla-La Mancha facturaron 555,04 millones de euros que, lo que supone el $48 \%$ del total regional, un peso importante, frente a las sociedades capitalistas, como señalan Bono et al. (2012).

El sector de cooperativas de vino está integrado por dos tipos de cooperativas. Por una parte un reducido número de ellas (10) concentran el mayor porcentaje de facturación (el $33 \%$ ) y, en el otro extremo, un elevado número de cooperativas de tamaño pequeño (Tabla 3). Esta atomización es típica del modelo mediterráneo y está provocada por múltiples factores, entre los que cabe destacar los de índole cultural, geográfico, razones de carácter social y político. El arraigo local y la dispersión geográfica han dificultado las fusiones y el crecimiento se ha apoyado en gran medida en la figura de la cooperativa de segundo grado (Baamonde, 2009).

En el año 2012, Baco se ha convertido en el primer grupo comercializador de España y Bodegas Virgen de las Viñas es la primera cooperativa de primer grado en facturación de vinos y mostos del país. En definitiva, el cooperativismo agrario constituye una importante realidad en la región, no sólo social, sino también económica, pues ha experimentado un importante desarrollo empresarial (Melián y Millán, 2007). 
Tabla 1. Posición del vino de Castilla-La Mancha en el año 2012

Table 1. The magnitude of wine of Castilla La Mancha in 2012

\begin{tabular}{lccc}
\hline & Producción (HI) & Área (Ha) & Exportación (HI) \\
\hline Unión Europea-27 & 156.150 .160 & 3.009 .500 & 50.123 .306 \\
España & 38.583 .000 & 943.000 & 24.652 .897 \\
Castilla-La Mancha & 18.622 .280 & 442.292 & 11.595 .975 \\
Castilla-La Mancha/Unión europea-27 (\%) & 11,93 & 14,70 & - \\
Castilla-La Mancha/España (\%) & 48,27 & 46,90 & 47,03 \\
\hline
\end{tabular}

Fuente: Fondo Español de Garantía Agraria (2013) para la producción; MAGRAMA (2013) para el área; Observatorio Español del Mercado del Vino (2013) para las exportaciones.

Tabla 2. Número de sociedades cooperativas vino de Castilla-La Mancha y facturación (Millones $€$ ) de las sociedades cooperativas Table 2. Number of wineries from Castilla La Mancha and turnover (million $€$ ) of the cooperatives

\begin{tabular}{lcc}
\hline & España & Castilla-La Mancha \\
\hline $\mathrm{N}^{\circ}$ cooperativas & 628 & 218 \\
Facturación & & \\
$\quad$ Cooperativas $1^{\text {er }}$ Grado/SAT ${ }^{1}$ & 990,25 & 501,93 \\
$\quad$ Vino granel & 672,36 & 448,43 \\
$\quad$ Vino embotellado & 212,89 & 22,81 \\
Mostos & 45,63 & 28,72 \\
Alcoholes & 9,05 & 0,00 \\
Uva & 46,72 & 1,03 \\
Otros productos & 3,56 & 0,94 \\
Cooperativas 2 ${ }^{\circ}$ Grado & - & 53,09 \\
Vino granel & - & 35,52 \\
Vino embotellado & - & 0,00 \\
Mostos & - & 14,02 \\
Alcoholes & - & 2,11 \\
Uva & - & 1,44 \\
Otros productos & - & 53,09 \\
Empresas comercializadoras de vino & 5.520 & 1.148 \\
Industria alimentaria & 88.673 & 6.660 \\
\hline
\end{tabular}

${ }^{1}$ Sociedad Agraria de Transformación.

Fuente: Cooperativas Agro-alimentarias (2014) y MAGRAMA (2013). 
Tabla 3. Ranking de facturación de las principales cooperativas agrarias y grado de integración Table 3. Ranking by turnover of the main agricultural cooperatives and their degree of integration

\begin{tabular}{llccc}
\hline Ranking & Cooperativa & Grado & $\begin{array}{c}\text { Facturación } \\
\text { (Millones } € \text { ) }\end{array}$ & $\begin{array}{c}\text { Facturación respecto } \\
\text { al sector (\%) }\end{array}$ \\
\hline 1 & BACO & 2 & 38,58 & 7,0 \\
2 & Bodegas Virgen de las Viñas & 1 & 31,77 & 5,7 \\
3 & Bodegas Yuntero & 1 & 19,36 & 3,5 \\
4 & El Progreso & 1 & 18,07 & 3,3 \\
5 & Cristo de la Vega & 1 & 14,99 & 2,7 \\
6 & Bodegas Rezuelo & 1 & 13,80 & 2,5 \\
7 & S. Cristo del Espíritu Santo & 1 & 11,86 & 2,1 \\
8 & San Isidro & 1 & 11,75 & 2,1 \\
9 & Unión Campesina Iniestense & 1 & 11,51 & 2,1 \\
10 & Ntra. Sra. de Manjavacas & 1 & 10,84 & 2,0 \\
\hline & Total & 182,53 & 33 \\
\hline
\end{tabular}

Fuente: Cooperativas Agro-Alimentarias (2014).

Marco teórico y discusión de variables

Según la teoría neoclásica en una situación de equilibrio a largo plazo todas las empresas, consideradas como agentes económicos individuales y racionales, tienden a maximizar sus resultados a partir de una idéntica función de producción, obteniendo un beneficio extraordinario nulo. En este planteamiento teórico, la preocupación por conocer la configuración interna de tales agentes económicos es inexistente. Sin embargo, en la realidad los beneficios existen y se manifiestan en muy diferente medida entre las distintas unidades empresariales y de forma independiente de la actividad económica concreta que realicen. (Sainz, 2002). Por eso, el estudio de la rentabilidad de los productores de vino representa actualmente uno de los objetivos más importantes, con el fin de analizar y verificar el estado de este segmento
(Chinnici et al., 2013). Ritchie y Kolodinsky (2003) señalan la importancia de medir la rentabilidad como un componente básico de la evaluación de la eficacia global de una empresa. Bagnoli y Megali (2011) señalan que la medición del desempeño económico y financiero es utilizada por las cooperativas para comprobar su responsabilidad financiera.

Las medidas de desempeño económico, pueden obtenerse a partir del uso de diferentes variables cada una de las cuales proporciona información sobre aspectos concretos de la empresa y, por lo tanto, en este trabajo vamos a incluir varias medidas de desempeño económico, al objeto de recoger las distintas actuaciones de las bodegas. Con carácter general, las medidas de desempeño económico más utilizadas son VA (Valor añadido) (Fischer y Schornberg, 2007); el ROA (Rentabilidad Económica) y ROI (Rentabilidad Financiera). Jarvis et al. (2000) y Dewenter y Malatesta 
(2001) las utilizan el ámbito empresarial general; Schiefer y Hartmann (2008) para la industria agroalimentaria; Amadieu y Viviani (2010) para el sector vinícola francés; Gilinsky et al. (2010) para las empresas vinícolas de California, la India e Italia y Sellers-Rubio (2010), para la industria española del vino. Por último y dada la particularidad del desempeño del sector cooperativo, se ha incluido la variable Rentabilidad del Socio, medida como la ratio Compras/Ventas.

Si consideramos la Función Objetivo de las empresas, se observa como el objetivo de las empresas mercantiles es la maximización del beneficio, mientras que para el sector cooperativo es la maximización de la Renta del Socio. Ambos tipos de empresas, comparten cuando su tamaño es pequeño, el objetivo de Supervivencia. En este trabajo, se considera la existencia de dos tipos de rentas para el socio de la cooperativa. En primer lugar, una Renta de Capital, que se percibe vía beneficio y para la cual, son adecuados indicadores de rentabilidad y, en segundo lugar, la renta del socio vía precios de compra. Los socios de las bodegas cooperativas son proveedores de uva, por lo que una parte de las rentas que perciben los socios se recibe a partir del precio que la bodega paga por la uva. Desde un punto de vista contable, esto se recoge en la cifra de Compras, por lo que un alto precio pagado por la uva, es una mejora de renta de los socios. Para capturar esta Renta del Socio, se define el indicador Compras sobre Ventas. A mayor valor de este ratio, más elevada será la renta recibida por los socios cooperativistas (Simón, 1996).

Es necesario tener en cuenta, que de todas las medidas de desempeño consideradas, es la Rentabilidad del Socio, la que recoge de una manera más precisa la comprensión de las distintas actuaciones llevadas a cabo por las bodegas. El ROI, es una medida de rentabilidad que no es la más interesante para el sector cooperativo. En aquellos casos en los que sea necesario comparar bodegas con diferente estructura de propiedad del capital, será VA y ROA, las que reflejen la rentabilidad de producción, pero no podemos esperar resultados comparables en el ROI entre ambos tipos de empresas por la diferencia de objetivos. Para las empresas capitalistas la maximización del beneficio y para las empresas cooperativas, la maximización de la renta percibida por el socio. Por lo que la incorporación de esta medida particular de sector, se refleja como adecuada para comprender la evolución del sector cooperativo.

Identificar los factores que inciden en la competitividad de una empresa, al ser muchos y diversos, es tarea compleja (Sainz, 2002). Para Cuervo (1993) son determinantes del desempeño los recursos y habilidades que emplee la empresa. Es por ello que en este estudio se opta por relacionar el desempeño con variables de entorno, de las estrategias comerciales y financieras y ayudas públicas. A continuación, se presentan las variables utilizadas, autores que las emplean en el estudio del desempeño y el signo que las relaciona con él.

Las variables de entorno afectan al desempeño de las empresas y a su crecimiento $y$, en consecuencia, el modelo organizativo adoptado puede ser explicado en términos de entorno (Dobbs y Hamilton, 2007). Se utilizarán: la edad, el tamaño, la intensidad de capital y el riesgo estratégico. El signo esperado de la variable edad sobre el desempeño puede ser positivo o negativo. Gardebroek et al. (2010) señalan que la edad de la empresa está significativa e inversamente relacionada con la rentabilidad. Otros autores como Mata y Portugal (1994) indican una relación inversa con el riesgo de fracaso empresarial y una relación positiva con el desempeño (Sánchez y García-Pérez; 2003). En cuanto al tamaño, una gran cantidad de literatura ha identificado la influencia positiva del tamaño de la empresa en su rentabilidad (Lee y Xiao, 2011). Esto se puede atribuir al hecho de que 
sólo las empresas más grandes pueden conseguir economías de escala y, por lo tanto, reducir su coste medio por unidad de medida (García-Fuentes et al., 2012). Contrariamente, el trabajo de Das et al. (1998) encuentra una relación inversa entre tamaño y rentabilidad. En general, la literatura describe la intensidad del capital como un efecto positivo en el crecimiento de las empresas (Altinkemer et al., 2011). Mientras, Segarra y Teruel (2007), apuntan la mayor intensidad tecnológica e innovadora como una dificultad para las empresas de menor tamaño.

En cuanto al riesgo estratégico como señala Chou et al. (2009) la teoría de valoración de activos, como el modelo Sharpe-Lintner-Black, y la teoría de valoración de arbitraje de Ross, afirman una relación positiva entre el rendimiento y medida de riesgo. Sin embargo, Bowman (1980) identifica una relación negativa entre el riesgo y la rentabilidad media. Fiegenbaum (1990) señalan que existe una asociación negativa entre riesgo y rendimiento cuando una empresa tiene rendimientos por debajo de su nivel objetivo y una asociación positiva para las empresas con rendimientos por encima del objetivo. Un menor valor de la variable de desempeño implica un mayor riesgo. Por lo que el mayor riesgo estratégico, será el de las empresas cuyas medidas de desempeño estén en el cuartil primero, teniendo en cuenta su cálculo (ver Tabla 4).

Las estrategias comerciales están en el foco del desempeño de las bodegas. Ante un sector en claro declive en consumo, así como un aumento del número de países productores, el grado de rivalidad en el sector es muy alto e impone la adopción de estrategias comerciales que permitan dar salida al producto. Estas estrategias, no dependen solo de las decisiones de la empresa, sino que también se ven afectadas por las decisiones adoptadas por el resto de bodegas que operan en el sector, en un contexto globalizado. Se emplean como variables representativas de las estra- tegias comerciales: el crecimiento de las ventas, las exportaciones y la segmentación del mercado. Como señala Brush et al. (2000) la mayoría de las empresas valoran el crecimiento de las ventas. En su investigación, Brush et al. (2000), obtienen una relación significativa y positiva en su influencia sobre ROA.

Sobre el comportamiento de las exportaciones en la industria del vino Castaldi et al. (2003) y Maurel (2009) revelaron que las exportaciones tiene un impacto positivo en las empresas de este sector. Reijonen et al. (2012) afirman que la orientación al mercado son considerados como factores que mejoran el rendimiento de la empresa (Narver y Slater 1990; Wong y Merrilees 2008). Muchos estudios demuestran el efecto positivo de la segmentación del mercado sobre los resultados empresariales (Simpson y Taylor, 2002; Vorhies et al., 1999).

Las variables financieras en el sector del vino, están relacionadas con características especiales derivadas de su proceso de producción. Para las bodegas, no solo es importante el acceso a la financiación sino equilibrio corto/largo plazo de los fondos en base al plazo de devolución. Para este estudio se consideran: el endeudamiento, la liquidez, la solvencia y el flujo de caja.

Brush et al. (2000) y Rajan y Zingales (1995) señalan una relación negativa entre endeudamiento y rentabilidad, altos niveles de deuda no contribuyen a aumentar el rendimiento. Namiki (2013) señala que la liquidez tiene una influencia significativa y negativa en el desempeño de pequeñas empresas en la etapa inicial de una recesión, disminuyendo su efecto en la etapa posterior. Otros en cambio consideran que es un instrumento útil, junto con la liquidez y la capacidad de endeudamiento, para mejorar el rendimiento de las empresas (Bradley et al., 2011; Cheng y Kessner, 1997; Latham y Braun, 2008). Amendola et al. (2012) encuentran evidencia de que las 


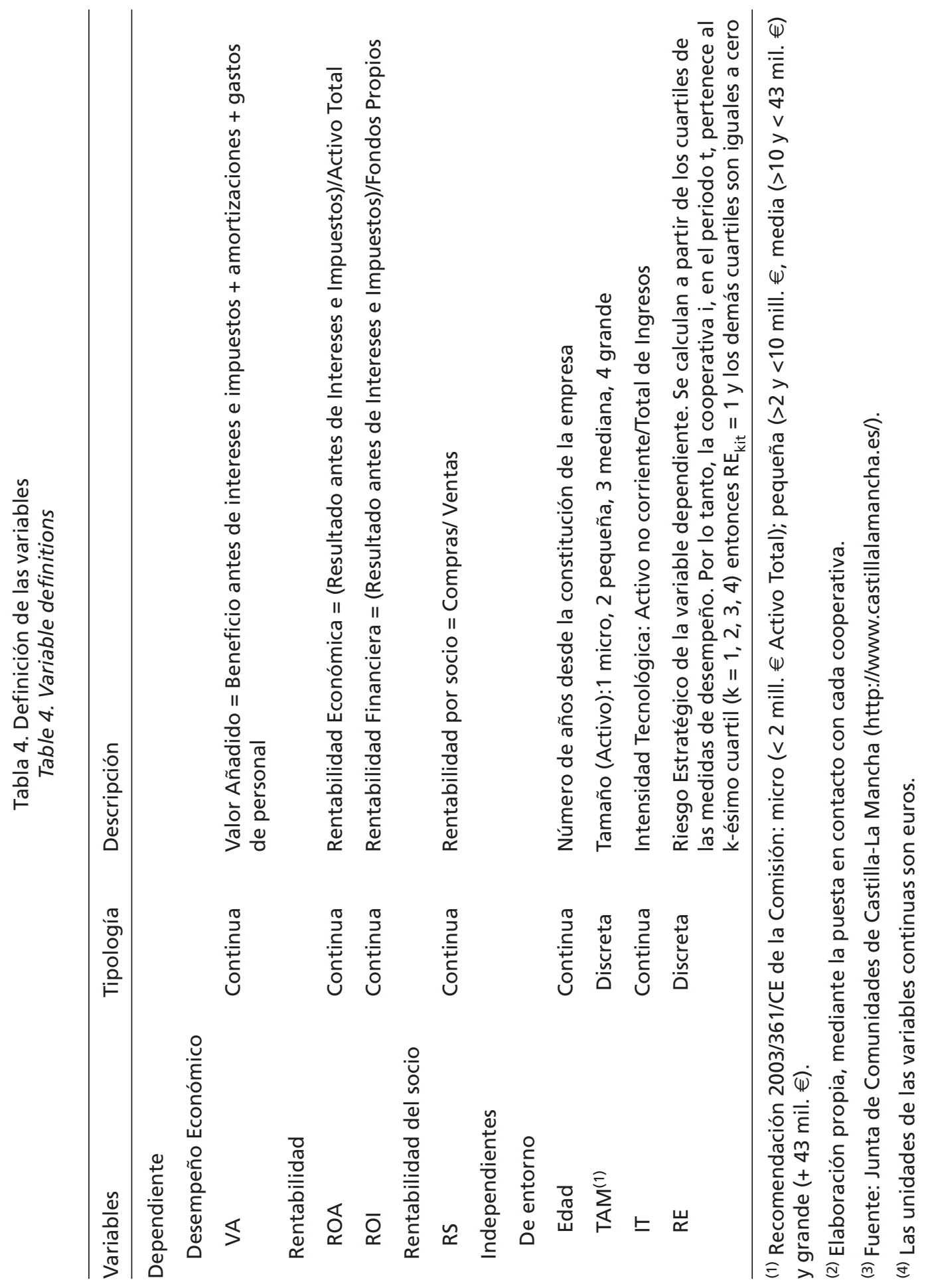




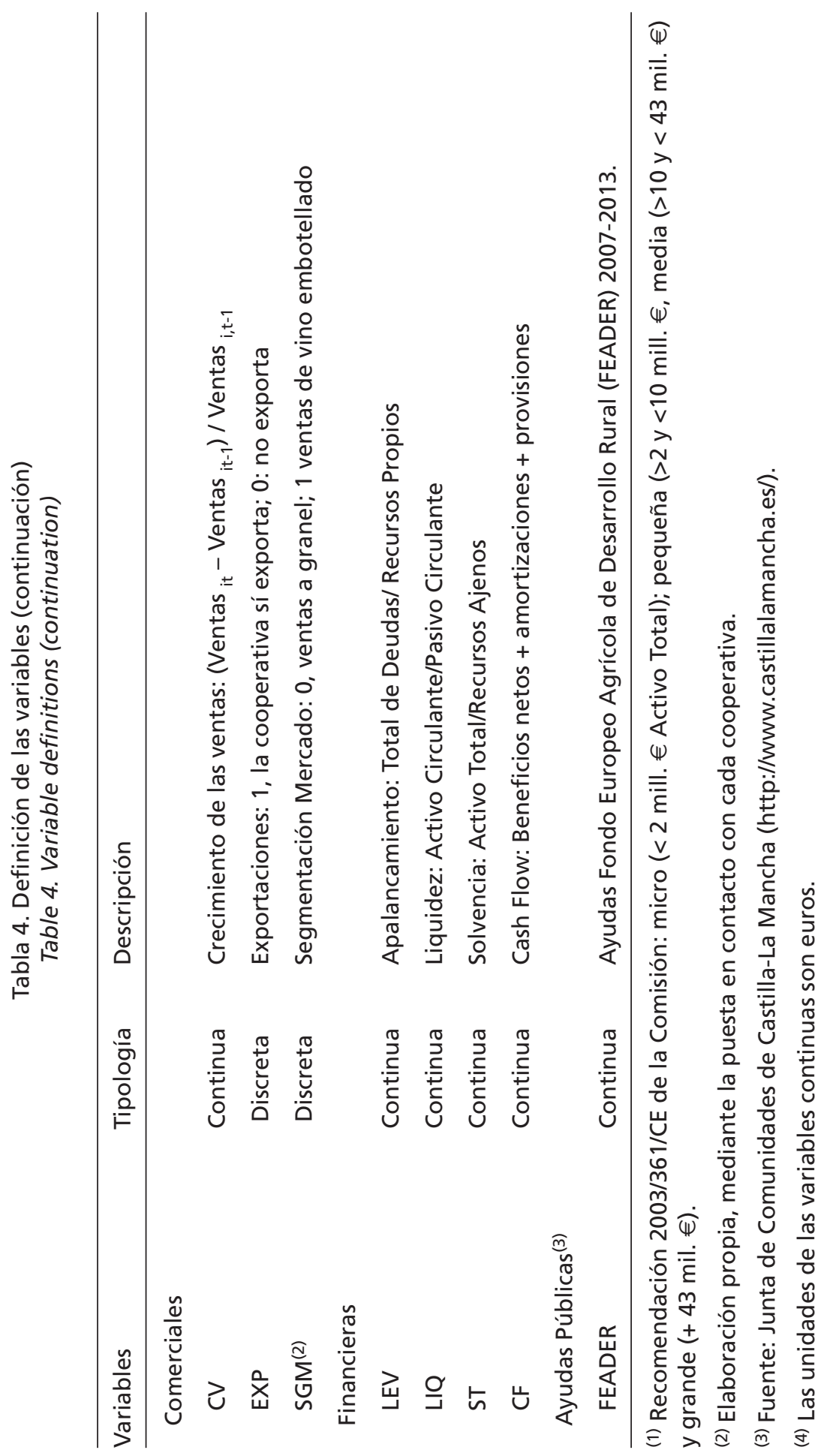


empresas de Italia con mayor garantía y solvencia y menos endeudados con los bancos tienen mayor índice de supervivencia. Görg y Spaliara (2008) concluyen lo mismo para las empresas de Reino Unido y Francia. El desempeño de las empresas también está estrechamente asociado a su capacidad de generar flujo de caja (Guariglia et al., 2011). Hong et al. (2012) señalan que, para las empresas de China, la relación entre el flujo de caja libre y el desempeño financiero es negativa, es decir, un exceso de flujo de caja libre liderará el rendimiento financiero a declinar. En cambio, Yungchih (2010) obtiene una relación positiva entre ROA y el flujo de caja para unas empresas de Taiwan

Las Ayudas Públicas, están afectando al desempeño del sector. Como señala Bono et al. (2012), las medidas de apoyo FEADER han contribuido al desarrollo de las cooperativas vitivinícolas sobre la base de las regiones de Veneto (Italia) y de Castilla-La Mancha (España).

\section{Material y Base de Datos}

Para el estudio empírico del trabajo, se ha tomado como Base de Datos, la compuesta por cooperativas cuya actividad es la elaboración de vino (código 1102 del Registro Nacional de Actividades Económicas de España 2009) que operan en Castilla-La Mancha, en el periodo 2002-2011. Los datos se han obtenido de los Registros Provinciales de cooperativas de la región. Este período corresponde, en ciclo de crecimiento económico (2002-2006) y período de crisis (2007-2011). Es una muestra, integrada en un $98 \%$ por empresas de menor tamaño (micro, $22 \%$, y pequeñas y medianas empresas, 76\%). Esta muestra no supone problemas de significatividad para los resultados, dado que el error muestral representa el 0,2\%, a un nivel de confianza del $95 \%$ según la fórmula de cálculo de error para poblaciones finitas, tomando como referencia la variable importe neto de la cifra de ventas para 2011.
Se conforma un panel no balanceado de datos para el período 2002-2011, en donde las variables especificadas para este estudio se recogen en la Tabla 4.

En la Tabla 5 se incluyen los estadísticos descriptivos de las variables. Como se puede observar, existe un rango muy amplio de variación de resultados, fundamentalmente por dos razones. En primer lugar, el periodo de tiempo analizado mezcla, una primera etapa hasta el año 2006 de crecimiento y bonanza económica, con un segundo periodo, a partir de 2007 de acusada crisis económica. En segundo lugar, hay que considerar las diferencias de tamaño de las cooperativas que forman parte de la base de datos, dado el elevado peso que las cooperativas de menor tamaño tienen en la base de datos objeto de estudio.

Al objeto de completar esta información y dada la disparidad de datos, se ha realizado el análisis Dupont de descomposición del beneficio (Figura 1). Mediante este análisis, se calcula el valor medio de los componentes de generación de valor para todo el periodo y para ambos sub-periodos. Después para establecer diferencias en las medias de ambos sub-periodos se aplicará el contraste no paramétrico de Mann-Whitney (Tabla 6). La razón ha sido el rechazo de la Hipótesis nula de Normalidad de las variables del análisis Dupont en la prueba de Kolmogorov-Smirnov, con p-valor inferior a 0,05 (Tabla 6).

Como se observa en dicha figura, las cooperativas de Castilla-La Mancha presentan valores de ROI negativos en el periodo de crecimiento y positivo en el de crisis $y$, además, existen diferencias significativas que explican su aumento, consecuencia de la reducción del apalancamiento financiero y del coste de la deuda.

Desde un punto de vista de proceso de producción, la caída de ROA es consecuencia de la caída de la rentabilidad de las ventas, que aparece reflejada en la caída del Valor Añadido 
Tabla 5. Estadísticos descriptivos de las variables Table 5. Descriptives of the variables

\begin{tabular}{|c|c|c|c|c|c|}
\hline Variables & $n$ & Mínimo & Máximo & Media & Desviación típica \\
\hline \multicolumn{6}{|c|}{ Dependientes } \\
\hline RS & 346 & 0,01 & 51,34 & 0,86 & 2,74 \\
\hline VA & 346 & -18462981 & 23779375 & 1636508 & 39149328 \\
\hline ROA & 346 & $-1,99$ & 10,21 & 0,14 & 0,64 \\
\hline ROI & 346 & $-16,464$ & 47,13 & 0,66 & 3,48 \\
\hline \multicolumn{6}{|c|}{ Independientes } \\
\hline \multicolumn{6}{|l|}{ Continuas } \\
\hline Edad & 346 & 0 & 96 & 46,02 & 21,10 \\
\hline IT & 346 & 0,05 & 46,59 & 0,83 & 2,41 \\
\hline $\mathrm{CV}$ & 316 & $-0,99$ & 11,72 & 0,11 & 0,78 \\
\hline LEV & 346 & $-84,07$ & 1516,8 & 7,0 & 82,98 \\
\hline LIQ & 346 & $-1,01$ & 28,47 & 1,34 & 1,86 \\
\hline ST & 346 & $-137,84$ & 36,33 & 1,78 & 8,08 \\
\hline $\mathrm{CF}$ & 346 & -18986786 & 23207212 & 1337311 & 3756403 \\
\hline \multirow[t]{2}{*}{ FEADER } & 346 & 0 & 811470 & 20195 & 90804 \\
\hline & \multicolumn{4}{|c|}{ Frecuencia } & \\
\hline Discretas & $(=0)$ & $(=1)$ & $(=2)$ & $(=3)$ & $(=4)$ \\
\hline TAM & & 89 & 197 & 54 & 6 \\
\hline RG(c4) & 260 & 86 & & & \\
\hline RG(c3) & 259 & 87 & & & \\
\hline $\mathrm{RG}(\mathrm{c} 2)$ & 259 & 87 & & & \\
\hline EXP & 147 & 199 & & & \\
\hline SGM & 340 & 6 & & & \\
\hline
\end{tabular}

RS: Rentabilidad por socio; VA: Valor añadido; ROA: Rentabilidad sobre Activos; ROI: Rentabilidad financiera; Edad: Años de constitución empresa; IT: Intensidad tecnológica; CV: Crecimiento ventas; LEV: Apalancamiento; LIQ: Liquidez; ST: Solvencia; CF: Tesorería; RG: Riesgo estratégico (en cada cuartil); EXP: Exportaciones; TAM: Tamaño; SGM: segmentación de mercado; FEADER: Fondo Europeo Agrario de Desarrollo Rural. 

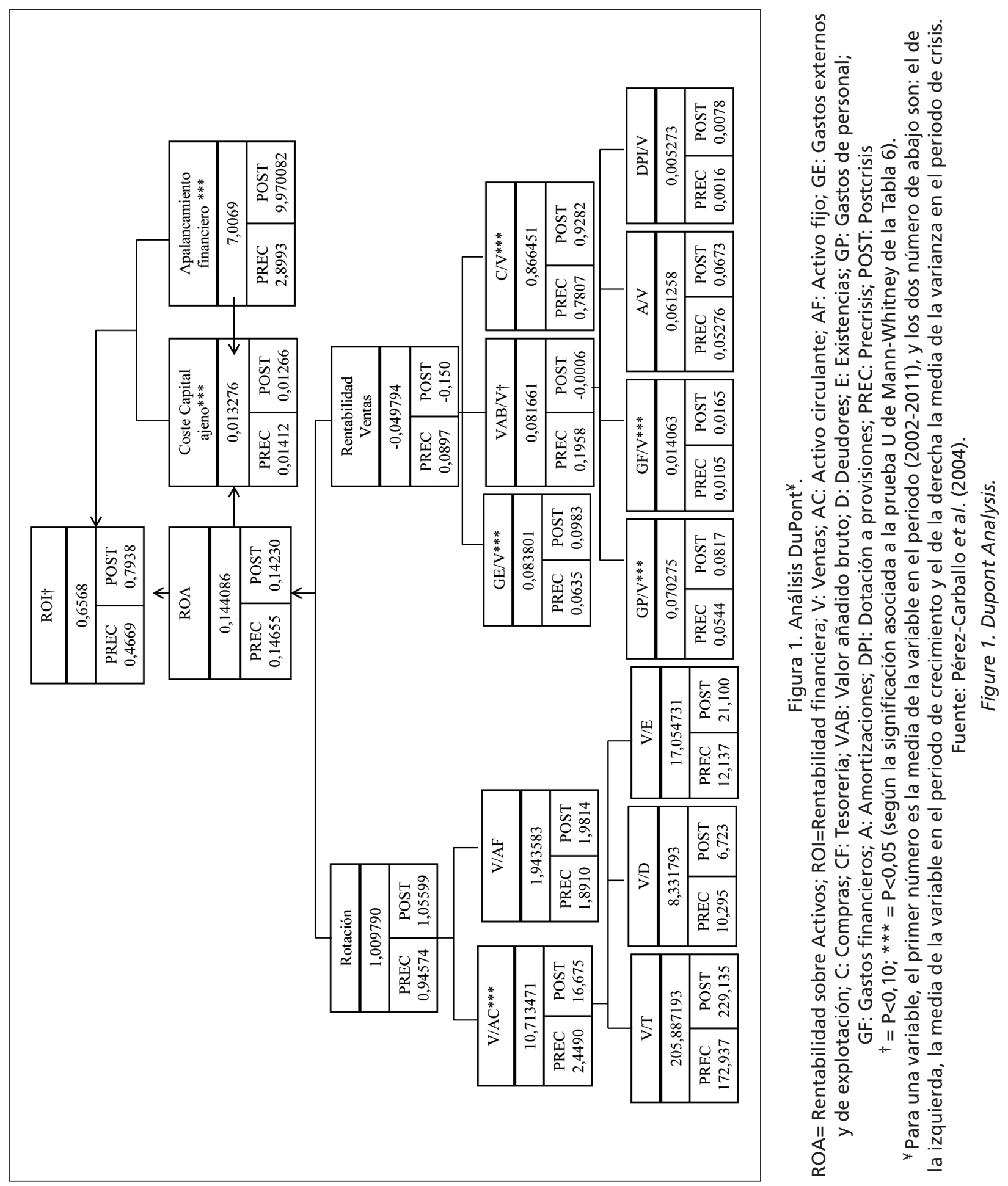
Tabla 6. Prueba de Kolmogorov-Smirnov y de de Mann-Whitney Table 6. Kolmogorv-Smirnov Test and Mann-Whitney Test

\begin{tabular}{lcccc}
\hline & \multicolumn{3}{c}{ Test } \\
\cline { 2 - 5 } & \multicolumn{3}{c}{ Kolmogorov-Smirnov ${ }^{1}$} & \multicolumn{1}{c}{ Mann-Whitney $^{2}$} \\
\cline { 2 - 5 } & Z & Sig. Asintót. ${ }^{3}$ & U & Sig. asintót. $^{3}$ \\
\hline Rentabilidad sobre activos (ROI) & 5,94 & 0 & 12718 & 0,04 \\
Rentabilidad financiera (ROA) & 5,05 & 0 & 13386 & 0,20 \\
Coste del capital ajeno & 8,55 & 0 & 11933 & 0,004 \\
Apalancamiento financiero & 8,47 & 0 & 11701 & 0,002 \\
Rotación & 3,83 & 0 & 13333 & 0,18 \\
Rentabilidad de las ventas & 8,05 & 0 & 13541 & 0,26 \\
Rotación activo circulante & 8,39 & 0 & 12805 & 0,05 \\
Rotación activo fijo & 3,65 & 0 & 14419 & 0,87 \\
GE /Nentas & 6,11 & 0 & 10338 & $4,0 \mathrm{e}^{-06}$ \\
Valor añadido bruto (VAB)/Ventas & 8,04 & 0 & 12897 & 0,07 \\
Compras/Ventas & 7,92 & 0 & 11449 & $7,0 \mathrm{e}^{-04}$ \\
Ventas/Tesorería & 6,83 & 0 & 12843 & 0,33 \\
Ventas/Existencias & 7,98 & 0 & 11937 & 0,42 \\
Ventas/Deudores & 6,35 & 0 & 12108 & 0,38 \\
Gastos Personal (GP)/Ventas & 6,26 & 0 & 9638 & $7,7 \mathrm{e}^{-08}$ \\
Gastos financieros (GF)/Ventas & 3,83 & 0 & 12631 & 0,03 \\
Activo/Ventas (A/V) & 5,00 & 0 & 13116 & 0,11 \\
Dotación a provisiones (DPI)/Ventas & 8,23 & 0 & 14502 & 0,91 \\
\hline
\end{tabular}

${ }^{1}$ Hipótesis Nula: el conjunto de datos siguen una distribución normal.

${ }^{2}$ Hipótesis Nula: no existen diferencias entre ambos subperiodos de tiempo.

${ }^{3}$ Significación asintótica (bilateral).

${ }^{4}$ Gastos externos y de explotación.

respecto a las ventas, así como el aumento del ratio compras sobre ventas. A este respecto señalar, que el aumento del ratio es consecuencia de una disminución de las ventas, no tanto de un aumento de los pagos de materia prima.

\section{Metodología}

Para el desarrollo del estudio se utilizó la técnica Pooled Cross Section Time Series como en los trabajos de Dewenter y Malatesta (2001), 
Goddard et al. (2004), Aparaschivei et al. (2011). En concreto, se manejó la corrección Driscoll y Kraay (1998). Las razones fueron, en primer lugar la mejor adecuación a los datos disponibles, al tratarse de un panel de datos no balanceado y segundo garantizar una inferencia estadística válida ante una varianza no constante $\sigma_{u}^{2} \neq c t e$, y una correlación serial de los errores $\operatorname{Cov}\left(u_{i}, u_{i}\right) \neq 0, \forall j=I$. Ante esta situación, como señala Hoechle (2007), las correcciones tradicionales resultan insuficientes. Driscoll y Kraay proponen una estimación de la matriz de varianzas y covarianzas de los parámetros estimados $\hat{\beta}$, que proporciona desviaciones típicas de robustas con heterocedasticidad y autocorrelación.
Así, para evaluar el efecto de los factores propuestos sobre el desempeño de la industria de elaboración de vino de Castilla-La Mancha y contrastar las hipótesis, se han planteado un modelo de Panel con la corrección de Driscoll y Kraay. La limitación principal que presenta este método es el no poder incluir términos fijos (ficticias) temporales y transversales con el fin de analizar el impacto concreto de los intervalos temporales y de los agentes económicos que intervienen.

Para explicar las diferencias de las variables de rendimiento entre los dos períodos: crecimiento y crisis, se estimaron dos modelos. Sustituyendo $X_{\text {kit }}$ por el conjunto de variables exógenas se obtiene:

$$
\begin{aligned}
& Y_{i t}=\sum_{k=0}^{K} \beta_{k} X_{k i t}+u_{i t}=\beta_{0} X_{o i t}+\beta_{1} E d a d_{i t}+\beta_{2} T A M_{i t}+\beta_{3} I T_{i t}+ \\
& \beta_{4} R G(c 4)_{i t}+\beta_{5} R G(c 3)_{i t}+\beta_{6} R G(c 2)_{i t}+\beta_{7} C V_{i t}+\beta_{8} E X P_{i t}+ \\
& \beta_{9} O R M_{i t}+\beta_{10} L E V_{i t}+\beta_{11} H F_{i t}+\beta_{12} S V_{i t}+\beta_{13} C F_{i t}+\beta_{14} F E A D E R_{i t}+u_{i t}
\end{aligned}
$$

con $\mathrm{t}=2002,2003,2004,2005,2006$ modelo bonanza y $t=2007,2008,2009$ y 2011 modelo crisis; con i = 1, 2, ..., 42.

La variable $Y_{i t}$ asume los valores de rentabilidad socio (RS), valor añadido (VA), rentabilidad económica (ROA) y rentabilidad financiera (ROI), en la expresión (1). Las variables independientes manejadas son diversas y se han clasificado en cinco grupos: 1 . Variables de entorno: edad, tamaño (TAM), intensidad tecnológica (IT) y riesgo estratégico (RG(ck)), donde $k=1,2,3$, 4, son los cuartiles de la variable $Y_{i t}$ ). 2. Variables comerciales: tasa de crecimiento de las ventas (CV), exportaciones (EXP) y segmentación del mercado (SGM). 3. Variables financieras: apalancamiento (LEV), liquidez (LIQ), solvencia (ST) y flujo de caja (CF). 4. Ayudas Públicas de los Fondos europeos (FEADER).
La selección del método de estimación requiere tomar en consideración la naturaleza de la base de datos: 1) es un panel no balanceado porque no todas las cooperativas tienen observaciones en el periodo estudiado; 2) en el modelo se introducen variables exógenas ficticias (exportaciones, segmentación del mercado y los riesgos estratégicos) y 3) es un panel heterogéneo en el tiempo y en la sección cruzada. Este último punto lo identificamos con las pruebas de Wald para heterocedasticidad y la prueba Wooldridge para la autocorrelación ${ }^{1}$ (Tabla 7). Por todas estas razones se utiliza la corrección de Driscoll y Kraay ya que este método corrige la estimación de los errores estándar de forma tal que sean consistentes con heterocedasticidad, autocorrelación y paneles no balanceados.

1. La prueba de correlación contemporánea no se pudo calcular por ser un papel altamente no balanceado. 
Tabla 7. Prueba de Wald y Prueba de Wooldridge Table 7. Wald Test and Wooldridge Test

\begin{tabular}{lcccc}
\hline & \multicolumn{2}{c}{ Prueba Wald } & \multicolumn{2}{c}{ Prueba Wooldridge } \\
\cline { 2 - 5 } & chi2 (31) & Prob>chi2 & $\mathrm{F}(1,23)$ & Prob > F \\
\hline Pre-crisis & & & & \\
$\quad$ Valor añadido (VA) & $9,5 \mathrm{e}^{+29}$ & 0,0000 & 4,48 & 0,045 \\
Rentabilidad sobre Activos (ROA) & $8,4 \mathrm{e}^{+31}$ & 0,0000 & 2,88 & 0,10 \\
Rentabilidad financiera (ROI) & $2,1 \mathrm{e}^{+30}$ & 0,0000 & 2,62 & 0,12 \\
Rentabilidad por socio (RS) & $1,9 \mathrm{e}^{+34}$ & 0,0000 & 2,71 & 0,11 \\
Crisis & & & & \\
Valor añadido (VA) & $3,1 \mathrm{e}^{+30}$ & 0,0000 & 27,87 & 0,0000 \\
Rentabilidad sobre Activos (ROA) & $9,9 \mathrm{e}^{+4}$ & 0,0000 & 0,91 & 0,3450 \\
Rentabilidad financiera (ROI) & $1,7 \mathrm{e}^{+35}$ & 0,0000 & 14,26 & 0,0005 \\
Rentabilidad por socio (RS) & $3,7 \mathrm{e}^{+31}$ & 0,0000 & 1,24 & 0,2713 \\
\hline
\end{tabular}

\section{Resultados y discusión}

Los resultados de estos análisis se recogen en la Tabla 8 y sobre ellos, se pueden extraer las siguientes apreciaciones a propósito del desempeño de las bodegas cooperativas.

Las variables de entorno, presentan el comportamiento esperado en las hipótesis teóricas. Así, la variable edad, no tiene relevancia en la explicación del desempeño económico de las cooperativas; al mismo resultado para pequeñas empresas llegan Begley y Boyd (1986), y Acar (1993). En el caso de la rentabilidad socio, son las cooperativas más antiguas las que están trasladando una mejor rentabilidad al socio, para ambas etapas del ciclo económico. El tamaño, está relacionado positivamente con el indicador de desempeño medido en valor absoluto (VA). Sin embargo, en el momento en el que se pone en referencia a través de medidas de rentabilidad (ROA y ROI), su impacto es negativo. Es decir, altos volúmenes de producción y venta no ga- rantizan buenas medidas de desempeño relativo; sobre todo en empresas pequeñas como señalan Sánchez y García-Pérez (2003). Fu et al. (2002) llegan a la misma conclusión en un estudio para 1.276 pequeñas empresas de Taiwán. Si observamos cómo afecta el tamaño de la cooperativa a la Rentabilidad del Socio, los resultados difieren dependiendo del periodo analizado. Así, en periodos de bonanza y crecimiento, las bodegas cooperativas de mayor tamaño han proporcionado una mejor rentabilidad para el socio que las pequeñas. Sin embargo, en periodo de crisis, estas no pueden mantener esta rentabilidad ante la caída de ventas y por lo tanto, ofrecen peores parámetros de retribución a los socios que las bodegas de menor tamaño. Posiblemente, sea consecuencia de los gastos fijos se las bodegas de mayor tamaño. La intensidad tecnológica resulta significativa pero con signo negativo para el desempeño económico, en consonancia de lo que apuntan Segarra y Teruel (2007), por los obstáculos a los 


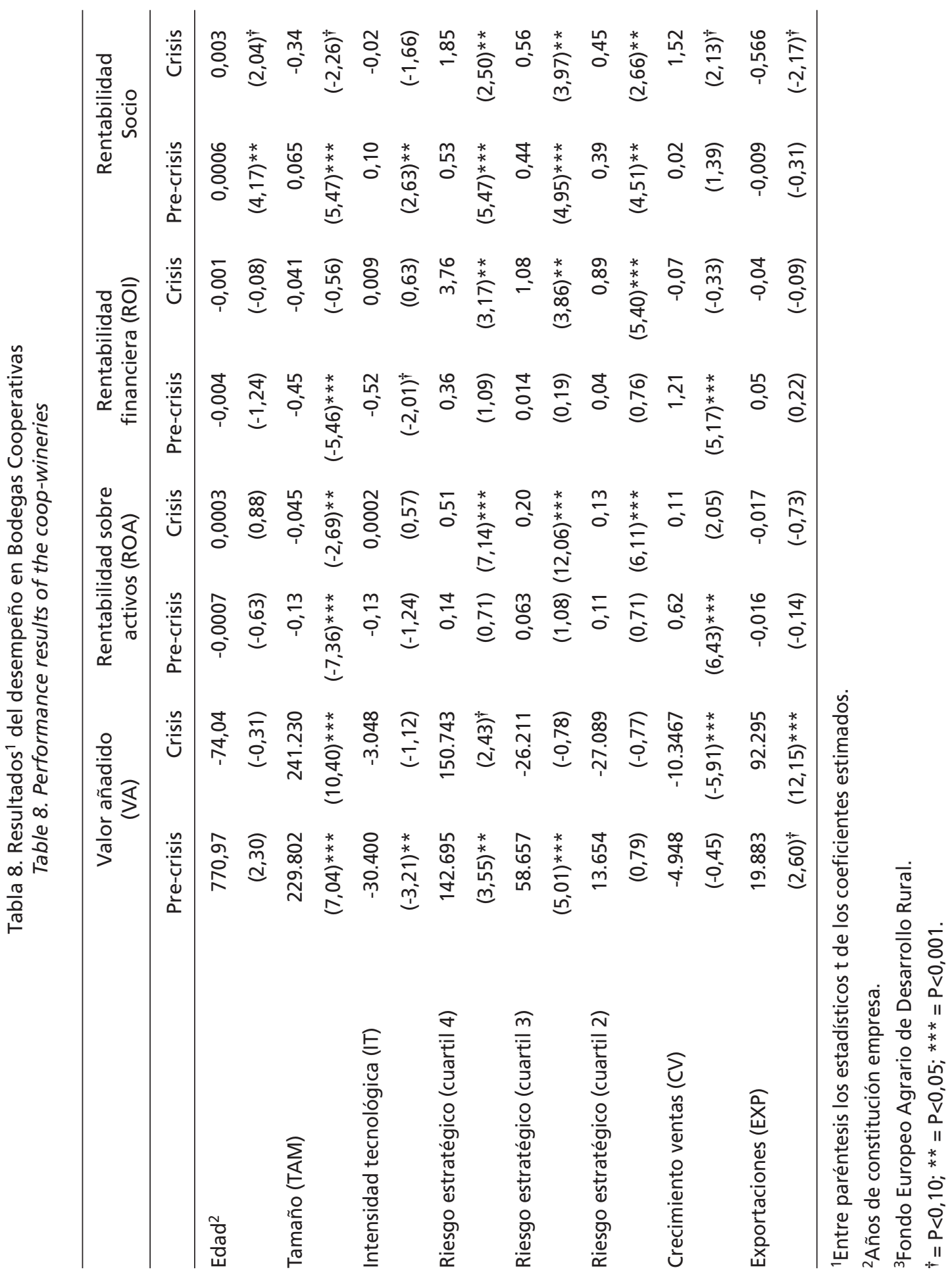




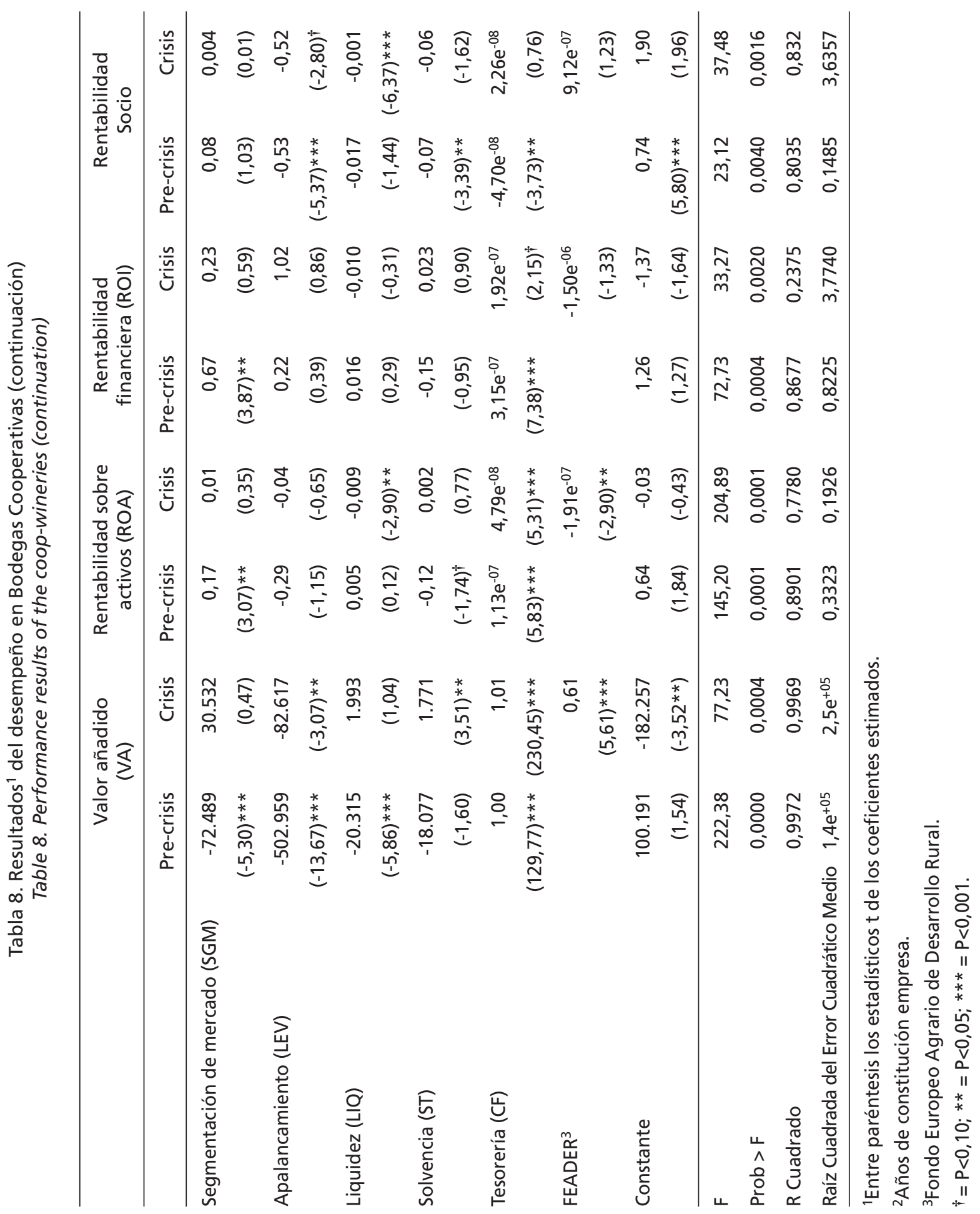


que se enfrentan las empresas de menor tamaño, en el acceso a fuentes de financiación. Señalar que en el periodo de bonanza económica, la intensidad tecnológica ha afectado positivamente a la rentabilidad del socio, esto puede ser consecuencia de las inversiones en modernización de las instalaciones acometidas por estas bodegas. Aun así, en periodo de crisis, son las bodegas cooperativas las que presentan relaciones positivas entre la rentabilidad del socio y la intensidad tecnológica. El riesgo estratégico de las cooperativas nos indica que a menor desempeño más riesgo, en todas las medidas para el periodo anterior a la crisis. Sin embargo, en el periodo de crisis, esta relación no se cumple, lo que indica que se están produciendo evoluciones no previstas en la medición del parámetro rentabilidad-riesgo. La Rentabilidad del Socio, es la única medida de desempeño que mantiene la coherencia entre rentabilidad-riesgo en ambos periodos analizados. Es importante señalar, que la ausencia de coherencia entre la relación rentabilidad-riesgo, podría estar indicando la existencia de Paradoja de Bowman, resultados ya encontrados en otros trabajos (Gschwandtner, 2012). Es decir, que la Rentabilidad del Socio, captura a través de esta medida las decisiones de rentabilidad / riesgo realizadas por las bodegas, cumpliendo el postulado, por el que a mayor rentabilidad mayor riesgo (medido como dispersión del mismo) y, por lo tanto, no existen comportamientos anómalos en la toma de decisiones.

El comportamiento de las variables comerciales, está siendo más errático si se compara los periodos de bonanza y crisis económica. El crecimiento de las ventas afecta de manera desigual a las distintas medidas de desempeño, ya que presenta resultados positivos en el periodo pre-crisis para el ROA y ROI y tan solo mantiene esta tendencia en el periodo de crisis para la Rentabilidad del Socio, lo que indica que el aumento en la cifra de ventas, se traslada de forma efectiva a los socios, incluso en momentos de crisis económica. Esto viene avalado por los resultados recogidos en el informe de la Cooperativas Agro-alimentarias (2014), en el que el sector cooperativo agroalimentario de Castilla-La Mancha está demostrando su robustez para aguantar la crisis.

Las exportaciones son un elemento positivo para la comercialización de producto en un momento de sobreoferta y caída de consumo de vino. Sin embargo, si consideramos el impacto que esta variable tiene en las medidas de desempeño se observa, como este es negativo. Esto nos indica que el esfuerzo comercializador en términos de producción, no se ve reflejado en el desempeño económico (signo negativo). Esto implica que en el momento en el que remonte la actividad económica, las bodegas cooperativas deberán realizar un importante esfuerzo para manteniendo la comercialización en niveles de producción, trabajar una mejora de precios que lleve a la mejora del desempeño económico de las mismas.

La segmentación al mercado tan solo ha permitido obtener impacto positivo en el desempeño en los periodos de bonanza económica, no así en el periodo de crisis. Es necesario tener en cuenta, el importante peso que las ventas de vino a granel representan en el sector cooperativo, ya que en el $98 \%$ de las cooperativas de la muestra las ventas de vino a granel superan las de vino embotellado. $Y$ dado que el precio medio de venta del granel es inferior al vino embotellado, esta situación afecta de forma negativa al desempeño de las bodegas. En el periodo de crisis, las bodegas cuya orientación de mercado ha sido el embotellado, han conseguido trasladar una mejora a la rentabilidad del socio, aunque con un impacto reducido sobre la misma. Este comportamiento, junto con el de las exportaciones nos indica la necesidad de realizar un importante esfuerzo en política de precios.

Las variables financieras, son clave para comprender las estrategias adoptadas por las bo- 
degas y como su situación financiera les permite adaptarse a las distintas fases del ciclo económico.

El nivel de endeudamiento de las cooperativas se mantiene en el periodo de crisis, lo que ha supuesto una reducción en el desempeño, es en este caso donde la Rentabilidad del Socio, actúa como amortiguador entre la política de recursos propios y recursos ajenos. Este balanceo entre ambos tipos de recursos, explica el comportamiento errático de las variables de solvencia total y flujo de caja, ya que se producen decisiones de traspasos de fondos en base al acceso al mercado de capitales, consecuencia de la restricción financiera en el periodo de crisis económica, coincidiendo con los resultados obtenidos por Namiki (2013), en empresas pequeñas y en plena recesión. Por último, se obtienen los mismos resultados que en Bono et al. (2012) respecto al impacto positivo de las medidas de apoyo FEADER, ya que estas han contribuido al desarrollo de las cooperativas vitivinícolas de Castilla-La Mancha.

En conclusión, el impacto que el ciclo de crisis está teniendo en la actividad económica está siendo muy relevante y también afecta de forma importante a las bodegas cooperativas de Castilla-La Mancha. En este trabajo, se ha analizado cual está siendo la respuesta de este sector ante un periodo largo de recesión, más de 7 años.

De los resultados obtenidos a partir del análisis de los estados financieros de las cooperativas vitivinícolas, se puede observar cómo están coexistiendo dos tipos de estrategias para intentar la supervivencia de las empresas y la transmisión de rentas a los socios cooperativistas. Por una parte y desde el periodo de bonanza económica, la modificación de la regulación comunitaria (Reglamento (CE) $n^{\circ}$ $1698 / 2005$ ) ha supuesto un cambio en las estrategias comerciales adoptadas por las bodegas. En este periodo las bodegas han realizado un notable esfuerzo comercial, tanto en crecimiento de ventas, exportaciones, como segmentación del mercado hacia el embotellado. Esta decisión les ha permitido colocar importantes volúmenes de producción que de otra forma hubieran sido destinados a destilación. Esta política, para que fuera posible implicó una estrategia de precios muy agresiva, que como se ve en los resultados no está afectando todo lo positivo que hubiera sido deseable en las medidas de desempeño. Sin embargo, se puede afirmar que esto mismo es lo que les está permitiendo sobrevivir en un periodo de crisis. Las cooperativas se han adaptado a la nueva situación, mostrando su viabilidad en el largo plazo. En el contexto comercial las exportaciones han sido un recurso en el periodo de crisis, aunque el vino a granel sigue orientado a su mercado.

Indudablemente, conforme crezca la actividad económica, las bodegas deberán realizar un importante esfuerzo en configurar una nueva política de precios, pero ya en un mercado donde la producción se está asentando.

En el ámbito financiero, las cooperativas de Castilla-La Mancha muestran una aceptable salud financiera que se ha mantenido en el periodo de crisis. Al contrario de los resultados obtenidos por Carmona et al. (2013), que aprecia un empeoramiento de la situación financiera, para el conjunto de sociedades cooperativas españolas. Por último, señalar que las ayudas FEADER para la mejora de las infraestructuras de las cooperativas han resultado significativas y propiciado el proceso de adaptación y de redefinición de las estrategias. En comparativa con las sociedades capitalistas, en un estudio centrado también en Castilla-La Mancha (Simón et al., 2014), las mayores diferencias se encuentran en una estructura financiera mucho más dañada, por el efecto crisis, en las sociedades capitalistas que en las cooperativas. Como estrategias comerciales, las exportaciones en las cooperativas han resultado más dinámicas que en las sociedades capitalistas. 


\section{Agradecimientos}

Los autores agradecen la financiación para esta investigación del Proyecto Regional de Castilla-La Mancha (PPII10-0158-0569), financiado con los Fondos FEDER de la Unión Europea, y del Proyecto del Plan Nacional de I+D+i, del Ministerio de Ciencia e Innovación (ECO2010-21242-C03-03).

\section{Bibliografía}

Acar AC (1993). The impact of key international factors on firm performance: an empirical study of small Turkish firms. Journal of Small Business Management 31(4): 86-91.

Altinkemer K, Ozcelik Y, Ozdemir ZD (2011). Productivity and performance effects of business process reengineering: a firm-level analysis. Journal of Management Information Systems 27(4): 129-161.

Amadieu P, Viviani JL (2010). Intangible effort and performance: the case of the French wine industry. Agribusiness 26(2): 280-306.

Amendola A, Ferragina AM, Pittiglio R, Reganati $F$ (2012). Are exporters and multinational firms more resilient over a crisis? First evidence for manufacturing enterprises in Italy. Economics Bulletin 32(3): 1914-1926.

Aparaschivei L, Vasilescu MD, Câtâniciu N (2011). The impact of investments and gross value added upon earnings. Theoretical and Applied Economics XVIII 2(555): 207-218.

Arcas-Lario N, Martín-Ugedo JF, Mínguez-Vera A (2013). Satisfacción de los socios con las cooperativas agrarias. Una explicación a partir de la Teoría de los Derechos de Propiedad. Información Técnica Económica Agraria 109 (4): 443-457.

Baamonde E (2009). El cooperativismo agroalimentario. Mediterráneo Económico 15: 229-246.

Bagnoli L, Megali C (2011). Measuring performance in social enterprises. Nonprofit and Voluntary Sector Quarterly 40(1): 149-165.
Begley TM, Boyd DP (1986). Executive and corporate correlates of financial performance in smaIler firms. Journal of Small Business Management 24(2): 8-15.

Bono P, Castillo Valero JS, Iliopoulos C (2012). Case study report; Structure and strategy of wine cooperatives: comparing Veneto, Italy to CastillaLa Mancha, Spain. Disponible en http://edepot. wur.nl/244926 (13 marzo 2014).

Bowman EH (1980) A risk/return paradox for strategic management. Sloan Management Review 21: 17-31.

Bradley SW, Shepherd DA, Wiklund J (2011). Swinging a double-edged sword: The effect of slack on entrepreneurial management and growth. Journal of Business Venturing 26(5): 537-554.

Brush T, Bromiley P, Hendrickx M (2000). The free cash flow hypothesis for sales growth and firm performance. Strategic Management Journal 21: 455-472.

Carmona P, Martínez J, Pozuelo J (2013). Diagnostico económico-financiero de la empresa cooperativa. (Un estudio comparado de los años 2004 y 2007). REVESCO 110: 43-95.

Castaldi RM, Sengupta S, Silverman M (2003). Improving export performance: the case of the US wine industry. Journal of Global. Marketing. 17(1): 45-56.

Cheng JLC, Kessner IF (1997). Organizational slack and responses to environmental shifts: the impact of resource allocation patterns. Journal of Management 23: 1-18.

Chinnici G, Pecorino B, Rizzo M, Rapisarda P (2013). Evaluation of the performances of wine producers in Sicily. Food Safety Management 14(35): 108-113.

Chou PH, Chou RK, Ko KC (2009). Prospect theory and the risk-return paradox: some recent evidence. Review of Quantitative and Finance and Accounting 33: 193-208.

Cooperativas Agro-alimentarias (2014). Estudio sectorial del cooperativismo agroalimentario de Castilla-La Mancha. Disponible en http:// www.agroalimentariasclm.coop/ (20 enero 2014). 
Cuervo A (1993). El papel de la empresa en la competitividad. Papeles de Economía Española 56: 363-378.

Das S, Sen PK, Sengupta S (1998). Impact of strategic alliances on firm valuation. Academy of Management Journal 41(1): 27-41.

Dewenter KL, Malatesta PH (2001). State-owned and privately owned firms: an empirical analysis of profitability, leverage, and labor intensity. The American Economic Review 91(1): 320-334.

Díaz-Barceló A (2007). Un análisis financiero comparado de cooperativas vitivinícolas de Castilla La Mancha con las empresas de los principales países productores de la Unión Europea. Universidad Politécnica de Madrid. Tesis Doctoral.

Dobbs M, Hamilton RT (2007). Small business growth: recent evidence and new directions. International Journal of Entrepreneurial Behaviour \& Research 13(5): 296-322.

Driscoll J, Kraay AC (1998). Consistent covariance matrix estimation with spatially dependent data. Review of Economics and Statistics 80: 549560.

Fiegenbaum A (1990). Prospect theory and the risk-return association: an empirical examination in 85 industries. Journal of Economic Behavior and Organization 14: 187-203.

Fischer C, Schornberg S (2007): Assessing the competitiveness situation of EU food and drink manufacturing industries: An index-based approach. Agribusiness 23(4): 473-495.

FEGA (2013). Fondo Español de Garantía Agraria. Disponible en: http://www.fega.es/PwfGcp/es/

Fu TW, Ke MC, Huang YS (2002). Capital growth, finance source and profitability of small businesses: evidence from Taiwan small enterprises. Small Business Economics 18(4): 257-267

García-Fuentes PA, Ferreira G, Lynn P (2012). Economic performance of U.S. multinational agribusinesses: foreign direct investment and firm strategy. Agribusiness 29(2): 242-255.

Gardebroek C, Turi KN, Wijnands JHM (2010). Growth dynamics of dairy processing firms in the European Union. Agricultural Economics 41: 285-291.
Gilinsky A, Lopez RH, Santini C, Eyler R (2010). Big bets, small wins? Entrepreneurial behavior and ROI. International Journal of Wine Business Research 22(3): 238-250.

Goddard J, Molyneux P, Wilson J (2004). The profitability of European banks: a cross-sectional and dynamic panel analysis. The Manchester School 72(3): 363-381.

Görg H, Spaliara ME (2008). Financial health, exports, and firm survival: A comparison of British and French firms. WP 2008 - 18. Loughborough University. $38 \mathrm{pp}$.

Gschwandtner A (2012). Evolution of profit persistence in the USA: Evidence from three periods. The Manchester School 80(2): 172-209.

Guariglia A, Liu X, Song L (2011). Internal finance and growth: microeconometric evidence on Chinese firms. Journal of Development Economics 96: 79-94.

Guzmán I, Arcas N, Ghelfi R, Rivaroli S (2009). Technical efficiency in the fresh fruit and vegetable sector: a comparison study of Italian and Spanish firms. Fruits 64(4): 1-10.

Hoechle D (2007). Robust standard errors for panel regressions with cross-sectional dependence. The Stata Journal 7(3): 281-312.

Hong Z, Shuting Y, Meng Z (2012). Relationship between free cash flow and financial performance evidence from the listed real estate companies in China. IPCSIT 36: 331-335.

Jarvis R, Curran J, Kitching J, Lightfoot G (2000). The use of quantitative and qualitative criteria in the measurement of performance in small firms. Journal of Small Business and Enterprise Development 7(2): 123-134.

JCCM (2014). http://www.castillalamancha.es/clm/ economia/unaregionquetrabajayemprende

Kalogeras N, Pennings J, Benos T, Doumpos M (2013). Which cooperative ownership model performs better? A financial-decision aid approach. Agribusiness 29(1): 80-95.

Latham S, Braun M (2008). The performance implications of financial slack during economic recession and recovery: observations from the software industry (2001-2003). Journal of Managerial Issues 20(1): 30-52. 
Lee $S$, Xiao Q (2011). An examination of the curvilinear relationship between capital intensity and firm performance for publicly traded U.S. hotels and restaurants. International Journal of Contemporary Hospitality Management 23(6): 862-880.

MAGRAMA (2013). Ministerio de Agricultura, Alimentación y Medio Ambiente, http://www.magrama.gob.es/es

Mata J, Portugal P (1994). Life duration of new firms. Journal of Industrial Economics 42: 227-246.

Maurel C (2009). Determinants of export performance in French wine SMEs. International Journal of Wine Business Research 21(2): 118-142.

Melián A, Millán G. (2007). El cooperativismo vitivinícola en España. Un estudio exploratorio en la denominación de origen de Alicante. REVESCO 93: 39-67.

Namiki N (2013). The performance implications of financial slack and its reduction during the great recession: the case of small-sized Japanese electronics companies. The Clute Institute: 188-196.

Narver JC, Slater SF (1990). The effect of a market orientation on business profitability. Journal of Marketing 54 (4): 20-35.

Observatorio Español del Mercado del Vino (2013). Disponible en: http://www.oemv.es/esp/-oemv.php

Pérez-Carballo A, Pérez-Carballo J y Vela E (2004): Principios de gestión financiera de la empresa. Ed. Alianza Universidades.

Rajan R, Zingales L (1995). What do we know about capital structure? Some evidence from international data. Journal of Finance, American Finance Association 50(5): 1421-60.

Reijonen H, Laukkanen T, Komppula R, Tuominen $S$ (2012). Are growing SMEs more market-oriented and brand-Oriented? Journal of Small Business Management 50(4): 525-716.

Ritchie WJ, Kolodinsky RW (2003). Nonprofit organization financial performance measurement. An evaluation of new and existing financial performance measures. Nonprofit Management \& Leadership 13: 367-381.

Ruíz AR (2013). El viñedo en espaldera: nueva realidad en los paisajes vitivinícolas de Castilla-La Mancha. Boletín de la Asociación de Geógrafos Españoles 63: 249-270.
Sainz A (2002). Análisis de los factores explicativos del éxito empresarial: una aplicación al sector de denominación de origen calificada Rioja. Universidad de La Rioja. Servicio de Publicaciones. Tesis Doctoral.

Sánchez JP, García-Pérez D (2003). Influencia del tamaño y la antigüedad de la empresa sobre la rentabilidad: un estudio empírico. Revista de Contabilidad 6(129): 169-206.

Schiefer J, Hartmann M (2008). Determinants of competitive advantage for Germanfood processors. Agribusiness 24(3): 306-319.

Segarra AB, Teruel MC (2007). Creación y supervivencia de las nuevas empresas en las manufacturas y los servicios. Economía Industrial 363: 47-58.

Sellers-Rubio R (2010). Evaluating the economic performance of Spanish wineries. International Journal of Wine Business Research 22(1): 73-84.

Simón K, Castillo JS, García MC (2014). Economic performance and the crisis: strategies adopted by the wineries of Castilla-La Mancha (Spain). Agribusiness. doi: 10.1002/agr.21392

Simón, K. (1996) Las cooperativas agrarias en Navarra: un enfoque empresarial. Ed. Caja Rural.

Simpson M, Taylor N (2002). The role and relevance of marketing in SMEs: towards a new model. Journal of Small Business and Enterprise Development 9: 370-382.

Vargas A, García E (2003). La medición del desempeño de las sociedades cooperativas agrarias. Perspectiva de los directores-gerentes de las provincias de Huelva y Jaén. Revista economía pública, social y cooperativa 46: 85-116.

Vorhies GW, Harker M, Rao CP (1999). The capabilities and performance advantages of marketdriven firms. European Journal of Marketing 33: 1171-1202.

Wong H, Merrilees B (2008). The performance benefits of being brand oriented. Journal of Product \& Brand Management 17: 372-383.

Yungchih G (2010). The impacts of free cash flows and agency costs on firm performance. Journal of Service Science \& Management 3: 408-418.

(Aceptado para publicación el 17 de octubre de 2014) 